\title{
LAS PLANTAS VASCULARES Y VEGETACIÓN DE LA BARRANCA TEPECAPA EN EL MUNICIPIO DE TLAYACAPAN, MORELOS, MÉXICO
}

\author{
Rodrigo Alejandro Hernández-Cárdenas ${ }^{1,2}$, Rosa Cerros-Tlatilpa ${ }^{1}$ \\ y Alejandro Flores-Morales ${ }^{1}$
}

${ }^{1}$ Universidad Autónoma del Estado de Morelos, Laboratorio de Sistemática y Morfología, Facultad de Ciencias Biológicas, Avenida Universidad 1001, Colonia Chamilpa, 62209 Cuernavaca, Morelos, México.

${ }_{2}^{2}$ Autor para la correspondencia: ralejandrohc@gmail.com

\section{RESUMEN}

El área de estudio, la Barranca Tepecapa de aproximadamente $3.5 \mathrm{~km}$ de longitud, se ubica dentro de la zona núcleo "Las Mariposas" en el Corredor Biológico Chichinautzin del estado de Morelos. Se realizaron colectas periódicas durante un año y medio, de octubre 2009 a abril 2011. Se colectaron 1018 ejemplares de Pteridofitas y afines, Gimnospermas y Angiospermas, pertenecientes a 99 familias, 266 géneros y 434 especies. Las familias mejor representadas son: Asteraceae, Fabaceae, Poaceae, Orchidaceae, Pteridaceae, Solanaceae y Bromeliaceae. Asimismo, se registran y describen tres tipos de vegetación para el área de estudio. Dos géneros (Amyris y Dasylirion) y 16 especies son nuevos registros para el estado de Morelos.

Palabras clave: área natural protegida, bosque mesófilo de montaña, Chichinautzin, listado florístico, Morelos, nuevos registros.

\section{ABSTRACT}

The study area, the Tepecapa gully with an approximate length of $3.5 \mathrm{~km}$, is situated in the core zone of "Las Mariposas" in the Biological Corridor Chichinautzin in the state of Morelos. Periodic collections were made over a year and a half, from October 2009 to April 2011. We collected 1018 specimens of Pteridophytes and relatives, Gymnosperms, and Angiosperms, belonging to 99 families, 266 genera and 434 species. The families Asteraceae, Fabaceae, Poaceae, Orchidaceae, Pteridaceae, Solanaceae and Bromeliaceae are the best represented ones. Three types of vegetation are reported and described for the 
study area. Two genera (Amyris and Dasylirion) and 16 species are new records for the state of Morelos.

Key words: Chichinautzin, floristic list, montane rain forest, Morelos, new records, protected natural area.

\section{INTRODUCCIÓN}

El estado de Morelos posee diversas áreas naturales protegidas, una de ellas es el Corredor Biológico Chichinautzin (COBIO). El COBIO tiene una extensión de 65901 ha y presenta tres zonas núcleo: Chalchihuites, Chichinautzin-Quiahustepec y Las Mariposas (Anónimo, 1988); en esta última se encuentra el área de estudio.

Entre las contribuciones al conocimiento de las plantas vasculares del COBIO se pueden citar los estudios florísticos y de vegetación de Hernández (1945), Ramírez-Cantú (1949), Bonilla-Barbosa y Viana (1997), Cerros-Tlatilpa y EspejoSerna (1998), Vega et al. (2008), Pulido-Esparza et al. (2009), Flores-Castorena y Martínez-Alvarado (2011). En este último se reportaron 1265 especies de plantas vasculares para el COBIO.

Los estudios florísticos son importantes porque permiten conocer la diversidad de una región determinada, principalmente en lugares de difícil acceso o poco explorados. Por ejemplo, las barrancas de la zona núcleo de "Las Mariposas" son refugio de diversidad biológica y no han sido estudiadas. Por lo tanto, el objetivo de este trabajo fue elaborar el listado florístico de las plantas vasculares y describir la vegetación que crece en el área de estudio.

Área de estudio

En las regiones aledañas a la zona núcleo "Las Mariposas" se encuentran varias barrancas, entre éstas destaca por su tamaño la de Tepecapa, con aproximadamente 3.5 kilómetros de largo. El área de estudio se ubica entre las coordenadas $18^{\circ} 58^{\prime} 11.7^{\prime \prime}-18^{\circ} 56^{\prime} 57.28^{\prime \prime} \mathrm{N}$ y 9900'30.0"-9900'21.21" O, en el municipio de Tlayacapan (Fig. 1), dentro de la Cuenca del río Balsas, en la vertiente sur del Eje Volcánico Transversal, con un intervalo altitudinal que va de los 1400 a los $2100 \mathrm{~m}$.

Basado en datos de la estación agrometereológica de Tlayacapan, en la barranca convergen dos tipos de clima; 1) por arriba de los $1600 \mathrm{~m}$ el clima es semicálido, con temperatura media anual menor de $22{ }^{\circ} \mathrm{C}$ y la precipitación anual menor de 


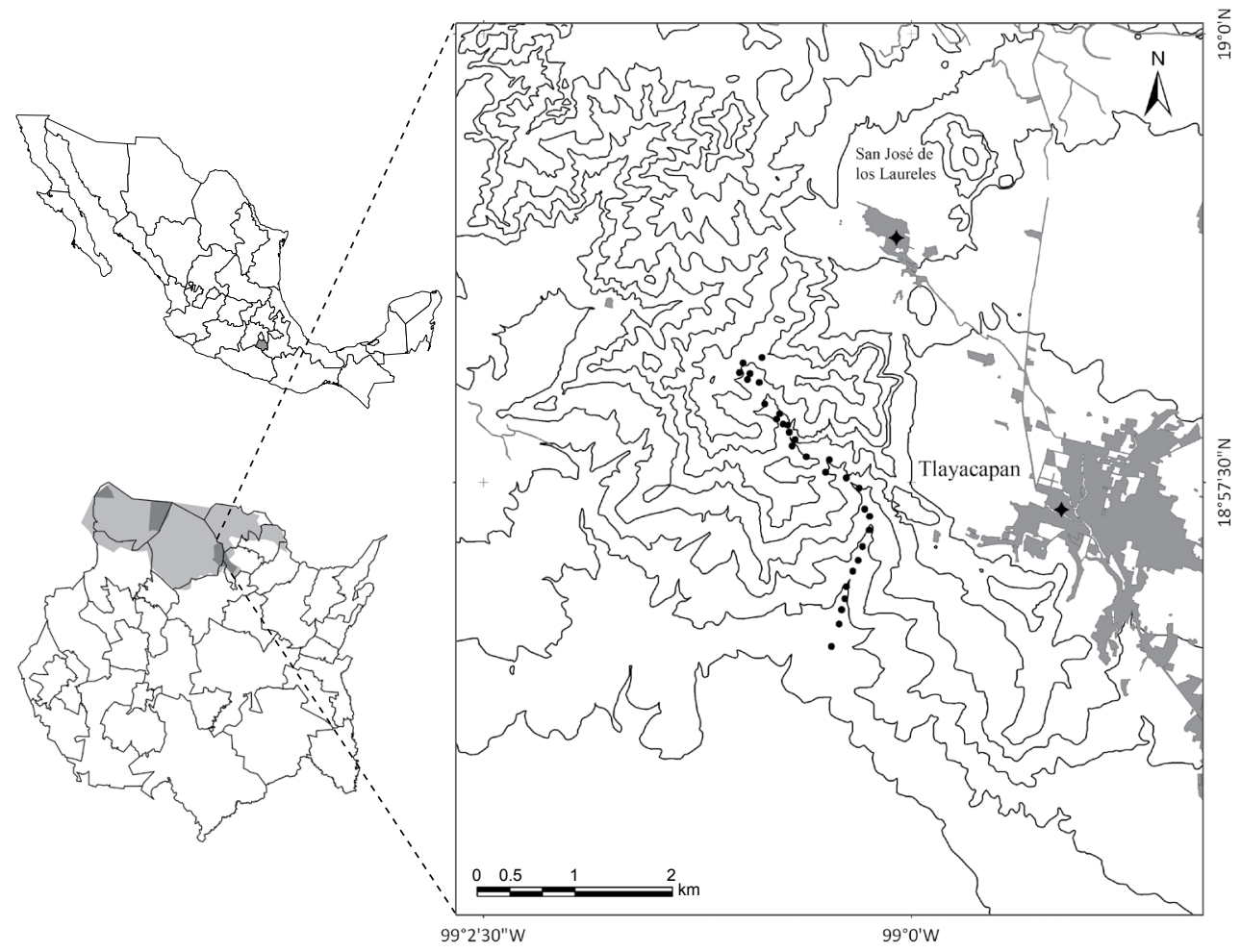

Fig. 1. Mapa del área de estudio de la Barranca Tepecapa, Tlayacapan, Morelos (los círculos negros señalan la ubicación de la barranca) proporcionado por el Laboratorio Interdisciplinario de Sistemas de Información Geográfica de la Universidad Autónoma del Estado de Morelos.

$1000 \mathrm{~mm}$, 2) por debajo de los $1600 \mathrm{~m}$ es cálido, con temperatura mayor de $22^{\circ} \mathrm{C}$ y la precipitación anual de 800 a $1000 \mathrm{~mm}$.

\section{MATERIALES Y MÉTODOS}

Para la elaboración del presente trabajo se realizaron de una a dos salidas al campo mensuales durante un año y medio (de octubre 2009 a abril 2011) y se colectaron 1018 ejemplares. La recolección y herborización de Pteridofitas, Gimnospermas y Angiospermas se siguió de acuerdo con Lot y Chiang (1986). La identificación de los ejemplares se llevó a cabo mediante el uso de claves y se cotejaron con los ejemplares de herbario (MEXU y UAMIZ). En casos especiales se contó 
con el apoyo de especialistas de las familias Bromeliaceae, Fabaceae, Fagaceae, Orchidaceae y Rutaceae. Para las Pteridofitas se siguió la clasificación de Mickel y Smith (2004) y para las plantas con flores la clasificación de Angiosperm Phylogeny Group III (2009). Para la caracterización de la vegetación se siguió a Rzedowski (2006) y León y Paniagua et al. (2010). Los ejemplares fueron depositados en los herbarios HUMO y UAMIZ.

\section{RESULTADOS}

Inventario florístico

Se identificaron 434 especies correspondientes a 266 géneros y 99 familias (Apéndice 1). En el Cuadro 1 se muestra el número de Pteridofitas y afines, Gimnospermas y Angiospermas (magnolides, monocotiledóneas y eudicotiledóneas). Las familias con el mayor número de géneros y especies respectivamente son (Cuadro 2): Asteraceae $(32,50)$, Fabaceae $(23,38)$, Poaceae $(18,27)$, Orchidaceae $(14,21)$ y Pteridaceae $(7,15)$. Los géneros con mayor número de especies son: Muhlenbergia (8), Tillandsia (8), Selaginella (8), Ipomoea (7), Cheilanthes (6), Desmodium (6), Salvia (6), Sedum (5), Dioscorea (5), Euphorbia (5), Quercus (5) y Oxalis (5).

Es importante destacar que a partir de este estudio los géneros Amyris y Dasylirion se registran por primera vez para el estado junto con 16 nuevos registros: $A s$ plenium sessilifolium (Aspleniaceae), Gnaphalium canescens (Asteraceae), Tillandsia supermexicana (Bromeliaceae), Clitoria mexicana y C. polystachya (Fabaceae), Dasylirion sp. (Nolinaceae), Randia obcordata (Rubiaceae), Amyris balsamifera y

Cuadro 1. Diversidad de familias, géneros y especies de la Barranca Tepecapa del municipio de Tlayacapan, Morelos.

\begin{tabular}{lccc}
\hline & Familias & Géneros & Especies \\
\hline Pteridofitas y afines & 10 & 21 & 43 \\
Gimnospermas & 1 & 1 & 1 \\
Angiospermas & & & \\
Magnolides & 2 & 3 & 6 \\
Monocotiledóneas & 16 & 58 & 95 \\
Eudicotiledóneas & 70 & 183 & 289 \\
\hline TOTAL & 99 & 266 & 434 \\
\hline
\end{tabular}


Cuadro 2. Familias mejor representadas en la Barranca Tepecapa del municipio de Tlayacapan, Morelos.

\begin{tabular}{lcc}
\hline Familia & Núm. de géneros & Núm. de especies \\
\hline Asteraceae & 32 & 50 \\
Fabaceae & 23 & 38 \\
Poaceae & 18 & 27 \\
Orchidaceae & 14 & 21 \\
Pteridaceae & 7 & 15 \\
Solanaceae & 6 & 14 \\
Bromeliaceae & 4 & 12 \\
Convolvulaceae & 3 & 11 \\
Rubiaceae & 6 & 10 \\
Acanthaceae & 7 & 8 \\
Commelinaceae & 4 & 8 \\
Onagraceae & 4 & 8 \\
Selaginellaceae & 1 & 8 \\
Apocynaceae & 6 & 7 \\
Crassulaceae & 2 & 7 \\
Lamiaceae & 2 & 7 \\
\hline
\end{tabular}

A. rekoi (Rutaceae), Sideroxylon cartilagineum (Sapotaceae), Brachistus stramoniifolius, Lycianthes peduncularis, L. pilosissima (Solanaceae), Selaginella hoffmannii y $S$. basipilosa (Selaginellaceae) y Phoradendron decipiens (Santalaceae).

La flora vascular del área de estudio está representada principalmente por cinco formas de vida: árboles, arbustos, hierbas, epífitas y parásitas. Las especies herbáceas predominan con 295 taxa (67\%), seguidas por los arbustos (61) (14\%), árboles (46) (11\%), epífitas (24) (6\%) y parásitas 8 (2\%). Los elementos arbóreos que resaltan a lo largo de la barranca son de las familias Burseraceae, Fabaceae, Fagaceae, Garryaceae, Meliaceae, Moraceae, Oleaceae, Pinaceae, Styracaceae y Symplocaceae. En el bosque de pino-encino (BPE) hay 363 especies, abundando las herbáceas con 275 taxa, los arbustos (38), los árboles (23), las epífitas (18) y las parásitas (7). Para el bosque mesófilo de montaña (BMM) se registran 80 especies, encontrando 26 taxa de plantas herbáceas, 15 spp. de arbustos, 21 spp. de árboles y 14 spp. de epífitas. Para el bosque tropical caducifolio (BTC) se reportan 98 especies, de las cuales destacan los elementos herbáceos (38), arbustos (22), árboles (20), epífitas (7) y una especie de parásita. 
Vegetación

En la barranca Tepecapa se reconocen tres tipos de vegetación: bosque mesófilo de montaña, bosque de pino-encino y bosque tropical caducifolio (Fig. 2).

Bosque mesófilo de montaña: El bosque mesófilo de montaña se desarrolla en un intervalo altitudinal de 1800 a $1950 \mathrm{~m}$. Fisonómicamente es un bosque con individuos de 5 a $30 \mathrm{~m}$ de altura. Se localiza en regiones de relieve accidentado y principalmente en laderas con pendiente pronunciada, así como zonas protegidas del viento y de la fuerte insolación.

Las especies de árboles que se pueden distinguir son: Ardisia compressa, Eugenia crenularis, Garrya longifolia, Guarea glabra, Meliosma dentata, Myrsine juergensenii, Fraxinus uhdei, Quercus candicans, Styrax ramirezii, Symplocos citrea, Ternstroemia lineata, Urera baccifera y Xylosma flexuosa. Algunos de los arbustos característicos son: Archibaccharis schiedeana, Brachistus stramoniifolius, Fuchsia arborescens, Monnina ciliolata, Montanoa frutescens, Oreopanax peltatus
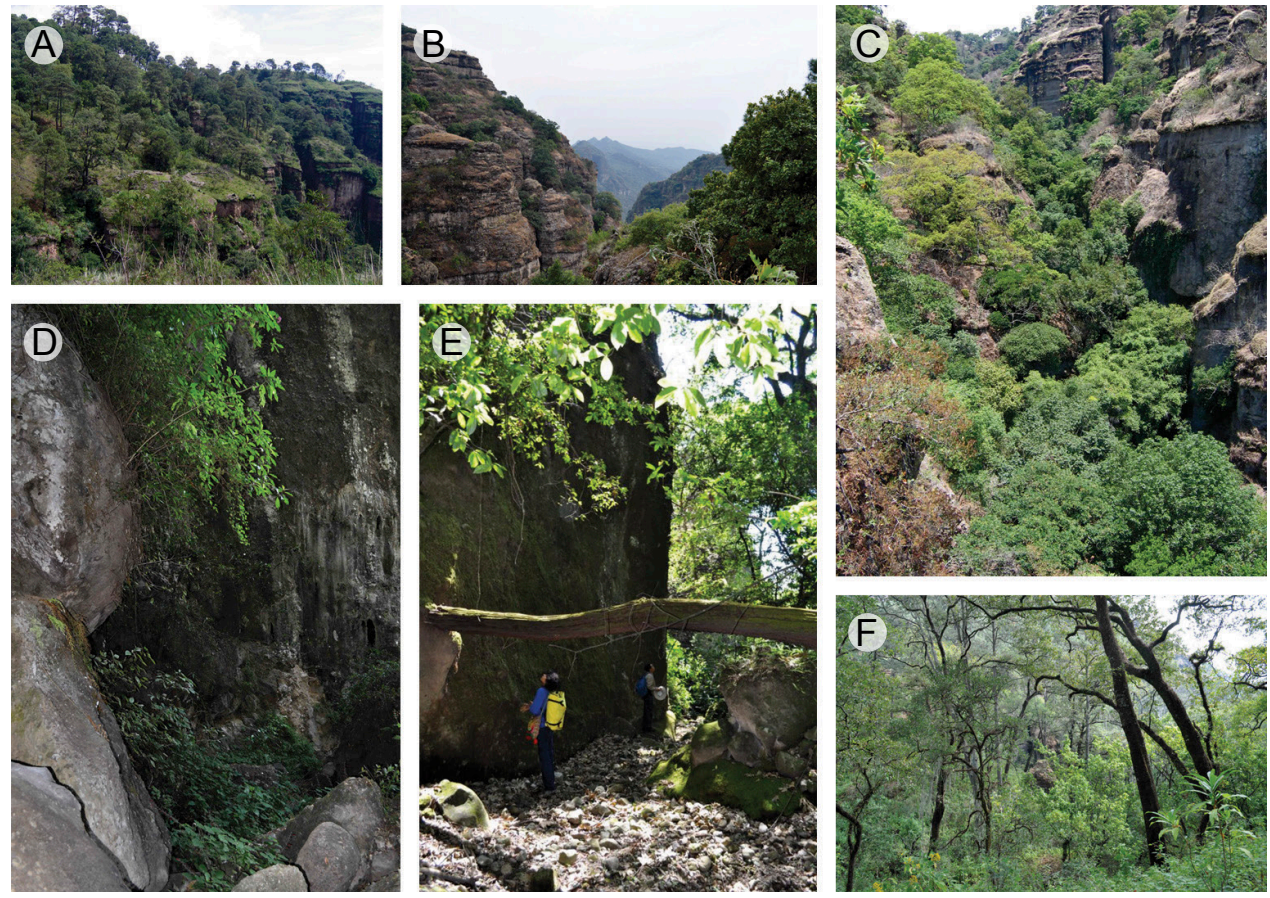

Fig. 2. A. Vista periférica al iniciar el descenso a la barranca; B. Cerro "Las Mariposas" y el inicio de la barranca; C. Parte norte de la barranca; D y E. Vista al interior de la barranca; F. Vista parcial del bosque mesófilo de montaña. 
y Piper amalago. En el estrato herbáceo son notables: Adiantum andicola, Arisaema macrospathum, Asplenium sessilifolium, Parietaria macrophylla, Periptera punicea, Pteris orizabae y Tournefortia hirsutissima. Entre las epífitas se observan: Campyloneurum angustifolium, Polypodium polypodioides, Tillandsia usneoides, Hintonella mexicana, Laelia autumnalis y Peperomia bracteata.

Bosque de pino-encino: El bosque de pino-encino prospera en altitudes de 1750 a $2100 \mathrm{~m}$. Son comunidades cuyos individuos miden de 10 a $30 \mathrm{~m}$ de alto. Se localiza en regiones planas o en laderas con pendientes pronunciadas. El estrato arbóreo está dominado por especies como: Acacia acatlensis, Ardisia compressa, Arbutus xalapensis, Lysiloma acapulcense, Pinus teocote, Quercus castanea, Q. glaucoides, Q. obtusata, $Q$. splendens, Ternstroemia lineata y Urera caracasana. En los componentes arbustivos destacan: Acalypha mollis, Agonandra racemosa, Buddleia sessiliflora, Erythrina breviflora, Eysenhardtia polystachya, Fuchsia arborescens, Lagascea rigida y Roldana angulifolia. Entre las herbáceas se distinguen: Arisaema macrospathum, Aristida schiedeana, Arracacia pubescens, Asplenium monanthes, Conopholis alpina, Dahlia coccinea, Deiregyne pyramidalis, Echeandia longipedicellata, Echinopepon gemellus, Geranium schiedeanum, Lobelia laxiflora, Lopezia miniata, Oplismenus burmannii, Sisyrinchium tenuifolium, Spigelia speciosa, Sprekelia formosissima, Verbesina tetraptera y Zeugites smilacifolius. Las epífitas están representadas por: Pecluma ferruginea, Phlebodium areolatum, Peperomia bracteata, Pleopeltis polylepis, Prosthechea linkiana y Tillandsia supermexicana.

Bosque tropical caducifolio: El bosque tropical caducifolio se desarrolla entre 1400 y $1750 \mathrm{~m}$. La altura de los árboles oscila entre 5 y $10 \mathrm{~m}$. Se localiza en regiones con poca pendiente. Algunas especies tienen tallos de color llamativo y superficie brillante, con cortezas exfoliantes y pierden sus hojas durante un periodo de 5 a 8 meses que corresponde a la época seca del año.

Dentro de las especies arbóreas se incluye a Bursera fagaroides, B. glabrifolia, B. longipes, Ficus cotinifolia, F. petiolaris, Lysiloma acapulcense y Trema micrantha. Las plantas arbustivas que sobresalen son: Brachistus stramoniifolius, Carlowrightia neesiana, Euphorbia schlechtendalii, Malvaviscus arboreus, Mimosa lacerata, Opuntia tomentosa, Pittocaulon praecox, Plumeria rubra, Randia thurberi y Roldana lobata. En el estrato herbáceo se observa a: Aeschynomene americana, Aristida schiedeana, Aster subulatus, Borreria prostrata, Cologania broussonetii, Diastatea tenera, Evolvulus alsinoides, Muhlenbergia ciliata, Sporobolus trichodes y Tagetes lunulata. Entre las plantas epífitas sobresalen: Tillandsia caput-medusae, T. hubertiana, T. recurvata, T. schiedeana y Trichocentrum cebolleta. 


\section{DISCUSIÓN}

En el presente estudio se destaca la alta diversidad florística de la barranca Tepecapa, ya que ésta concentra $56 \%$ de las familias, $27 \%$ de los géneros y $14 \%$ de los taxa pertenecientes al grupo de las Gimnospermas (17 especies, 5 géneros y 3 familias) y Angiospermas (3138 especies, 957 géneros, 173 familias) reportadas por Bonilla-Barbosa y Villaseñor (2003) para el estado de Morelos. Riba et al. (1996) citaron 21 familias, 50 géneros y 158 especies de Pteridofitas y afines para el estado, de las cuales solo 5 especies están reportadas para el municipio de Tlayacapan. La barranca posee $27.21 \%$ de las especies, $42 \%$ de los géneros y $47.61 \%$ de las familias mencionadas para Morelos.

Tepecapa concentra $34 \%$ de las 1265 especies de plantas vasculares registradas por Flores-Castorena y Martínez-Alvarado (2011) para el COBIO. Además, en el área de estudio se encontraron tres de las siete especies endémicas de monocotiledóneas del COBIO: Stelis nigriflora, Nemaconia dressleriana y Hechtia chichinautzensis (Pulido-Esparza et al., 2009; Martínez-Correa et al., 2010).

La barranca Tepecapa junto con los cerros "El Sombrerito" y "Las Mariposas" forman parte de la zona núcleo "Las Mariposas", y para estas dos últimas regiones se reportan 368 especies de plantas vasculares (Cerros-Tlatilpa y Espejo-Serna, 1998). Si consideramos la lista florística de Cerros-Tlatilpa y Espejo-Serna (1998), con la del presente estudio, se tiene un total de 743 especies, excluyendo aquellas que se comparten. Esto sugiere que en los cerros Las Mariposas, El Sombrerito y la barranca Tepecapa existe $58.73 \%$ de las especies vasculares reportadas para el COBIO.

En lo que concierne a vegetación, se reporta por primera vez en la barranca Tepecapa, municipio de Tlayacapan, el bosque mesófilo de montaña, ya que solo se conocía de los municipios de Huitzilac y Tepoztlán, estado de Morelos.

\section{CONCLUSIÓN}

Aunque la barranca Tepecapa se encuentra en un área poco accesible, no está exenta de la perturbación causada por actividades agrícolas, incendios y deforestación. Del mismo modo se enfrenta a la extracción selectiva de especies en la temporada navideña, por ejemplo: Agave dasylirioides, Dasylirion sp., Mammillaria spinosissima, Pinus teocote, Selaginella spp., y Tillandsia spp.

Finalmente, es necesario continuar con la exploración botánica y el estudio de la flora del Corredor Biológico Chichinautzin junto con otras áreas que no han sido 
estudiadas. Además, es importante fomentar en la población el manejo sustentable para la conservación de los recursos naturales.

\section{AGRADECIMIENTOS}

Se agradece al personal del Laboratorio Interdisciplinario de Sistemas de Información Geográfica de la Universidad Autónoma del Estado de Morelos la elaboración del mapa del área de estudio. A Jacqueline Ceja, Aniceto Mendoza, Adolfo Espejo, Ana Rosa López Ferrari, Alfonso Delgado, Susana Valencia, L. Torres Colín, Fernando Chiang y Oscar Dorado por la ayuda brindada en la identificación de algunos ejemplares. A los revisores anónimos por la revisión crítica del manuscrito y a los habitantes del pueblo de San José de los Laureles, municipio de Tlayacapan, por el permiso brindado para explorar sus tierras.

\section{LITERATURA CITADA}

Anónimo. 1988. Decreto por el que se declara el área de protección de la flora y fauna silvestre, ubicado en los municipios de Huitzilac, Cuernavaca, Tepoztlán, Jiutepec, Tlalnepantla, Yautepec, Tlayacapan y Totolapan, Morelos. Diario Oficial de la Federación. 30 de noviembre de 1988. México, D.F., México.

Angiosperm Phylogeny Group. 2009. An update of the Angiosperm Phylogeny Group classification for the orders and families of flowering plants: APG III. Bot. J. Linn. Soc. 161: 105-121.

Bonilla-Barbosa, J. R. y A. J. Viana. 1997. Listado florístico de México. XIV Flora del Parque Nacional Lagunas de Zempoala, México. Instituto de Biología, Universidad Nacional Autónoma de México. México, D.F., México. 31 pp.

Bonilla-Barbosa, J. R. y J. L. Villaseñor. 2003. Catálogo de la flora del estado de Morelos. Centro de Investigaciones Biológicas, Universidad Autónoma del Estado de Morelos. Cuernavaca, México. 129 pp.

Cerros-Tlatilpa, R. y A. Espejo-Serna. 1998. Contribución al estudio florístico de los cerros El Sombrerito y Las Mariposas (Zoapapalotl), municipio de Tlayacapan, Morelos, México. Polibotánica 8: 29-46.

Flores-Castorena, A. y D. Martínez-Alvarado. 2011. Sinopsis florística. Capítulo V. In: Bonilla-Barbosa, J., M. V. Mora, J. Luna-Figueroa, H. Colín y S. Santillán-Alarcón (eds.). Biodiversidad, conservación y manejo en el Corredor Biológico Chichinautzin condiciones actuales y prespectivas. Universidad Autónoma del Estado de Morelos. Cuernavaca, México. pp. 69-97.

Hernández, P. 1945. La flora maravillosa de Tepoztlán. Bol. Soc. Bot. Méx. 3: 13-15. 
León y Paniagua, L., I. Luna, M. A. Martínez y D. Tejero. 2010. VI Cuenca Alta del Balsas. In: Comisión Nacional para el Conocimiento y Uso de la Biodiversidad (ed.). El bosque mesófilo de montaña en México: amenazas y oportunidades para su conservación y manejo sostenible. Comisión Nacional para el Estudio de la Biodiversidad. D.F., México. pp. 88-97.

Lot, A. y F. Chiang (comps.). 1986. Manual de herbario. Administración y manejo de colecciones, técnicas de recolección y preparación de ejemplares de herbario. Consejo Nacional de la Flora de México, A.C. México, D.F., México. 142 pp.

Martínez-Correa, N., A. Espejo-Serna, A. R. López-Ferrari y I. Ramírez-Morillo. 2010. Two novelties in Hechtia (Bromeliaceae, Hechtioideae) from Mexico. Syst. Bot. 35: 745-754.

Mickel, J. T. y A. R. Smith. 2004. The pteridophytes of Mexico. Memoirs of the New York Botanical Garden 88: 1-1054.

Pulido-Esparza, V., A. Espejo-Serna y A. López-Ferrari. 2009. Las monocotiledóneas nativas del Corredor Biológico Chichinautzin. Acta Bot. Mex. 86: 9-38.

Ramírez-Cantú, D. 1949. Notas generales sobre la vegetación de la Sierra de Tepoztlán, Morelos. Anal. Inst. Biol. UNAM 20: 189-228.

Riba, R., L. Pacheco, A. Valdés e Y. Sandoval. 1996. Pteridoflora del estado de Morelos, México. Lista de familias, géneros y especies. Acta Bot. Mex. 37: 45-65.

Rzedowski, J. 2006. Vegetación de México. Comisión Nacional para el Conocimiento y Uso de la Biodiversidad. México, D.F., México. 504 pp.

Vega, G. A., J. López-García, D. L. Manzo. 2008. Análisis espectral y visual de vegetación y uso del suelo con imágenes Landsat ETM+ con apoyo de fotografías aéreas digitales en el Corredor Biológico Chichinautzin, Morelos, México. Invest. Geogr. 67: 59-75. 


\section{APÉNDICE 1}

Listado florístico de la Barranca Tepecapa en el municipio de Tlayacapan Morelos, México. Abreviaturas de vegetación: BMM, bosque mesófilo de montaña; BP-E, bosque de pino-encino y BTC, bosque tropical caducifolio. Forma de vida: $\mathrm{AR}$, árbol; $\mathrm{AB}$, arbusto; EPI, epífita; HER, herbácea; PAR, parásita. El asterisco (*) señala los nuevos registros para el estado.

\begin{tabular}{lll}
\hline Especie & Vegetación & $\begin{array}{r}\text { Forma } \\
\text { de vida }\end{array}$
\end{tabular}

\section{PTERIDOFITAS Y AFINES}

ASPLENIACEAE

Asplenium blepharophorum Bertol.

A. eatonii Davenp.

BP-E HER

A. monanthes L.

BP-E HER

*A. sessilifolium Desv.

BP-E HER

BLECHNACEAE

Blechnum occidentale L.

BMM HER

DRYOPTERIDACEAE

Dryopteris maxonii Underw. \& C. Chr.

D. rossii C. Chr.

BP-E HER

LOMARIOPSIDACEAE

Nephrolepis undulata (Afzel. ex Sw.) J. Sm.

POLYPODIACEAE

Campyloneurum angustifolium (Sw.) Fée

BP-E HER

BP-E HER

C. amphostenon (Kunze ex Klotzsch) Fée

Pecluma ferruginea (M. Martens \& Galeotti) M.G. Price

BMM, BP-E HER

Pecluma sp.

Phlebodium areolatum (Humb. \& Bonpl. ex Willd.) J. Sm.

Pleopeltis polylepis (Roem. ex Kunze) T. Moore

BMM EPI

BMM, BP-E EPI

BMM, BP-E EPI

BMM, BP-E EPI

BP-E HER

Polypodium polypodioides (L.) Watt

BMM, BP-E EPI

P. thyssanolepis A. Braun ex Klotzsch

BMM, BP-E EPI

PSILOTACEAE

BMM, BP-E EPI

Psilotum complanatum Sw.

BMM, BP-E HER

PTERIDACEAE

Adiantum andicola Liebm.

BP-E

HER

A. concinnum Humb. \& Bonpl. ex Willd.

BP-E HER

A. patens Willd.

BP-E HER

A. raddianum C. Presl

BP-E HER

Anogramma leptophylla (L.) Link

BP-E HER


Apéndice 1. Continuación.

\begin{tabular}{lcc}
\hline Especie & Vegetación & $\begin{array}{c}\text { Forma } \\
\text { de vida }\end{array}$ \\
\hline Argyrochosma pilifera (R.M. Tryon) Windham & BP-E & HER \\
Bommeria pedata (Sw.) E. Fourn. & BP-E & HER \\
Cheilanthes angustifolia Kunth & BP-E & HER \\
C. aurantiaca (Cav.) T. Moore & BP-E & HER \\
C. bonariensis (Willd.) Proctor & BP-E & HER \\
C. cuneata Kaulf. ex Link & BP-E & HER \\
C. kaulfussii Kunze & BP-E & HER \\
C. lendigera (Cav.) Sw. & BP-E & HER \\
Pellaea ternifolia (Cav.) Link & BP-E & HER \\
Pteris orizabae M. Martens \& Galeotti & BP-E & HER \\
SELAGINELLACEAE & & \\
*Selaginella basipilosa Valdespino & BP-E & HER \\
S. delicatissima Linden ex A. Braun & BP-E & HER \\
*S. hoffmannii Hieron. & BP-E & HER \\
S. landii Greenm. \& N. Pfeiff. & BP-E & HER \\
S. lepidophylla (Hook. \& Grev.) Spring & BP-E & HER \\
S. pallescens (C. Presl) Spring & BP-E & HER \\
S. schaffneri Hieron. & BP-E & HER \\
S. sellowii Hieron. & BP-E & HER \\
TECTARIACEAE & & \\
Ctenitis equestris (Kunze) Ching & BP-E & HER \\
WOODSIACEAE & & \\
Cystopteris fragilis (L.) Bernh. & BP-E & HER \\
Woodsia mollis (Kaulf.) J. Sm. & BP-E & HER
\end{tabular}

\section{GIMNOSPERMAS}

PINACEAE

Pinus teocote Schltdl. \& Cham.

BP-E

AR

\section{ANGIOSPERMAS}

MAGNOLIDES

HERNANDIACEAE

Gyrocarpus jatrophifolius Domin

PIPERACEAE

Peperomia ampla (Trel.) G. Mathieu

BMM HER

P. bracteata A.W. Hill

BMM, BP-E EPI

P. leptophylla Miq. 
Apéndice 1. Continuación.

\begin{tabular}{|c|c|c|}
\hline Especie & Vegetación & $\begin{array}{l}\text { Forma } \\
\text { de vida }\end{array}$ \\
\hline P. tetraphylla (G. Forst.) Hook. \& Arn. & BMM, BP-E & EPI \\
\hline Piper amalago L. & BMM & AR \\
\hline \multicolumn{3}{|l|}{ MONOCOTILEDÓNEAS } \\
\hline \multicolumn{3}{|l|}{ ASPARAGACEAE } \\
\hline Agave dasylirioides Jacobi \& C.D. Bouché & BP-E & HER \\
\hline A. horrida Lem. ex Jacobi & BP-E & HER \\
\hline Echeandia longipedicellata Cruden & BP-E & HER \\
\hline E. mexicana Cruden & BP-E & HER \\
\hline \multicolumn{3}{|l|}{ AMARYLLIDACEAE } \\
\hline Allium glandulosum Link \& Otto & BP-E & HER \\
\hline Manfreda pubescens (Regel \& Ortgies) Verh.-Will. ex Piña & BP-E & HER \\
\hline M. scabra (Ortega) McVaugh & BP-E & HER \\
\hline Sprekelia formosissima (L.) Herb. & BP-E & HER \\
\hline \multicolumn{3}{|l|}{ ALSTROEMERIACEAE } \\
\hline Bomarea hirtella (Kunth) Herb. & BP-E & HER \\
\hline \multicolumn{3}{|l|}{ ARACEAE } \\
\hline Arisaema macrospathum Benth. & BMM, BP-E & HER \\
\hline \multicolumn{3}{|l|}{ BROMELIACEAE } \\
\hline Hechtia chichinautzensis Martínez-Correa, Espejo \& López-Ferrari & BP-E & EPI \\
\hline Pitcairnia heterophylla (Lindl.) Beer & BTC & HER \\
\hline P. pteropoda L.B. Sm. & BP-E & HER \\
\hline Tillandsia caput-medusae E. Morren & BP-E, BTC & EPI \\
\hline T. hubertiana Matuda & BTC & EPI \\
\hline T. makoyana Baker & BTC & EPI \\
\hline T. recurvata (L.) L. & BP-E, BTC & EPI \\
\hline T. schiedeana Steud. & BTC & EPI \\
\hline *T. supermexicana Matuda & BP-E & EPI \\
\hline T. usneoides (L.) L. & BMM, BP-E & EPI \\
\hline Tillandsia $\mathrm{sp}$. & BP-E & EPI \\
\hline Viridantha atroviridipetala (Matuda) Espejo & BP-E & EPI \\
\hline \multicolumn{3}{|l|}{ COMMELINACEAE } \\
\hline Commelina coelestis Willd. & BP-E & HER \\
\hline C. diffusa Burm. f. & BP-E & HER \\
\hline C. leiocarpa Benth. & BP-E & HER \\
\hline C. tuberosa L. & BP-E & HER \\
\hline Thyrsanthemum macrophyllum (Greenm.) Rohweder & BP-E & HER \\
\hline Tinantia erecta (Jacq.) Schltdl. & BP-E & HER \\
\hline
\end{tabular}


Apéndice 1. Continuación.

\begin{tabular}{|c|c|c|}
\hline Especie & Vegetación & $\begin{array}{l}\text { Forma } \\
\text { de vida }\end{array}$ \\
\hline Tripogandra amplexans Handlos & BP-E & HER \\
\hline T. disgrega (Kunth) Woodson & BP-E & HER \\
\hline CYPERACEAE & & \\
\hline Carex longicaulis Boeck. & BP-E & HER \\
\hline Cyperus lanceolatus Poir. & BP-E & HER \\
\hline C. seslerioides Kunth & BP-E & HER \\
\hline C. spectabilis Link & BP-E & HER \\
\hline $\begin{array}{l}\text { Eleocharis acicularis (L.) Roem. \& Schult. } \\
\text { DIOSCOREACEAE }\end{array}$ & BP-E, BT-C & HER \\
\hline Dioscorea convolvulacea Schltdl. \& Cham. & BP-E & HER \\
\hline D. galeottiana Kunth & BP-E & HER \\
\hline D. lobata Uline & BP-E & HER \\
\hline D. ulinei Greenm. ex R. Knuth & BP-E & HER \\
\hline $\begin{array}{l}\text { D. urceolata Uline. } \\
\text { HYPOXIDACEAE }\end{array}$ & BP-E & HER \\
\hline $\begin{array}{l}\text { Hypoxis mexicana Schult. \& Schult. f. } \\
\text { IRIDACEAE }\end{array}$ & BP-E & HER \\
\hline Sisyrinchium tenuifolium Humb. \& Bonpl. ex Willd. & BP-E & HER \\
\hline $\begin{array}{l}\text { Tigridia meleagris (Lindl.) G. Nicholson } \\
\text { LEMNACEAE }\end{array}$ & BP-E & HER \\
\hline $\begin{array}{l}\text { Lemna aequinoctialis Welw. } \\
\text { LILIACEAE }\end{array}$ & BTC & HER \\
\hline $\begin{array}{l}\text { Calochortus cernuus J.H. Painter } \\
\text { NOLINACEAE }\end{array}$ & BP-E & HER \\
\hline $\begin{array}{l}\text { *Dasylyrion sp. } \\
\text { ORCHIDACEAE }\end{array}$ & BP-E & $\mathrm{AB}$ \\
\hline Bletia gracilis Lodd. & BP-E & HER \\
\hline B. neglecta Sosa & BP-E & HER \\
\hline B. purpurata A. Rich. \& Galeotti & BP-E & HER \\
\hline B. roezlii Rchb.f. & BP-E & HER \\
\hline Cypripedium irapeanum La Llave \& Lex. & BP-E & HER \\
\hline Deiregyne pyramidalis (Lindl.) Burns-Bal. & BP-E & HER \\
\hline D. rhombilabia Garay & BP-E & HER \\
\hline Habenaria crassicornis Lindl. & BP-E & HER \\
\hline H. novemfida Lindl. & BP-E & HER \\
\hline Hintonella mexicana Ames & BMM & EPI \\
\hline Laelia autumnalis (La Llave \& Lex.) Lindl. & BMM, BP-E & EPI \\
\hline
\end{tabular}


Apéndice 1. Continuación.

\begin{tabular}{|c|c|c|}
\hline Especie & Vegetación & $\begin{array}{l}\text { Forma } \\
\text { de vida }\end{array}$ \\
\hline Liparis greenwoodiana Espejo & BP-E & HER \\
\hline Malaxis brachyrrhynchos (Rchb. f.) Ames & BP-E & HER \\
\hline M. fastigiata (Rchb. f.) Kuntze & BP-E & HER \\
\hline M. lepidota (Finet) Ames & BP-E & HER \\
\hline $\begin{array}{l}\text { Nemaconia dressleriana (Soto Arenas) Van den Berg, Salazar \& } \\
\text { Soto Arenas }\end{array}$ & BMM, BP-E & HER \\
\hline Prosthechea linkiana (Klotzsch) W.E. Higgins & BMM, BP-E & EPI \\
\hline Schiedeella eriophora (Rob. \& Greenm.) Schltr. & BP-E & HER \\
\hline Stanhopea hernandezii (Kunth) Schltr. & BMM, BP-E & HER \\
\hline Stelis nigriflora (L.O. Williams) Pridgeon \& M.W. Chase & BP-E & HER \\
\hline $\begin{array}{l}\text { Trichocentrum cebolleta (Jacq.) M.W. Chase \& N.H. Williams } \\
\text { POACEAE }\end{array}$ & BTC & EPI \\
\hline Achnatherum constrictum (Hitchc.) Valdés-Reyna \& Barkworth & BP-E, BTC & HER \\
\hline Aristida schiedeana Trin. \& Rupr. & BP-E, BTC & HER \\
\hline Cenchrus ciliaris L. & BP-E & HER \\
\hline Digitaria ischaemum (Schreb.) Muhl. & BP-E & HER \\
\hline Enneapogon desvauxii P. Beauv. & BP-E & HER \\
\hline Melinis repens (Willd.) Zizka & BP-E & HER \\
\hline Microchloa kunthii Desv. & BP-E & HER \\
\hline Muhlenbergia ciliata (Kunth) Trin. & BP-E & HER \\
\hline M. diversiglumis Trin. & BP-E, BTC & HER \\
\hline M. implicata (Kunth) Trin. & BP-E & HER \\
\hline M. rigida (Kunth) Kunth & BP-E & HER \\
\hline M. robusta (E. Fourn.) Hitchc. & BP-E & HER \\
\hline M. tenella (Kunth) Trin. & BP-E & HER \\
\hline M. uniseta (Lag.) Columbus & BP-E & HER \\
\hline M. versicolor Swallen & BP-E & HER \\
\hline Oplismenus burmannii (Retz.) P. Beauv. & BP-E & HER \\
\hline Paspalum convexum Humb. \& Bonpl. ex Flüggé & BP-E & HER \\
\hline P. humboldtianum Flüggé & BP-E & HER \\
\hline P. melanospermum Desv. ex Poir. & BP-E & HER \\
\hline Schizachyrium sanguineum (Retz.) Alston & BP-E & HER \\
\hline Setaria parviflora (Poir.) Kerguélen & BP-E & HER \\
\hline Sporobolus trichodes Hitchc. & BP-E, BTC & HER \\
\hline Trachypogon spicatus (L. f.) Kuntze & BP-E & HER \\
\hline Triniochloa stipoides (Kunth) Hitchc. & BP-E & HER \\
\hline Tripsacum dactyloides (L.) L. & BP-E & HER \\
\hline
\end{tabular}


Apéndice 1. Continuación.

\begin{tabular}{|c|c|c|}
\hline Especie & Vegetación & $\begin{array}{l}\text { Forma } \\
\text { de vida }\end{array}$ \\
\hline Trisetum virletii E. Fourn. & BP-E, BTC & HER \\
\hline Zeugites smilacifolius Scribn. & BP-E & HER \\
\hline \multicolumn{3}{|l|}{ SMILACACEAE } \\
\hline Smilax moranensis M. Martens \& Galeotti & BMM, BP-E & HER \\
\hline \multicolumn{3}{|l|}{ EUDICOTILEDÓNEAS } \\
\hline \multicolumn{3}{|l|}{ ACANTHACEAE } \\
\hline Barleria micans Nees & BTC & $\mathrm{AB}$ \\
\hline Carlowrightia neesiana (Schauer ex Nees) T.F. Daniel & BTC & $\mathrm{AB}$ \\
\hline Dicliptera inaequalis Greenm. & BP-E & HER \\
\hline Dyschoriste hirsutissima (Nees) Kuntze & BTC & HER \\
\hline Justicia candicans (Nees) L.D. Benson & BMM, BTC & $\mathrm{AB}$ \\
\hline J. pringlei B.L. Rob. & BTC & $\mathrm{AB}$ \\
\hline Pseuderanthemum praecox (Benth.) Leonard & BP-E & HER \\
\hline Siphonoglossa mexicana Hilsenb. & BMM, BTC & $\mathrm{AB}$ \\
\hline \multicolumn{3}{|l|}{ AMARANTHACEAE } \\
\hline Iresine angustifolia Euphrasén & BP-E & HER \\
\hline I. diffusa Humb. \& Bonpl. ex Willd. & BMM & HER \\
\hline \multicolumn{3}{|l|}{ ANACARDIACEAE } \\
\hline Toxicodendron radicans (L.) Kuntze & BMM & $\mathrm{AB}$ \\
\hline \multicolumn{3}{|l|}{ APIACEAE } \\
\hline Donnellsmithia biennis (J.M. Coult. \& Rose) Mathias \& Constance & BP-E & HER \\
\hline D. mexicana (B.L. Rob.) Mathias \& Constance & BP-E & HER \\
\hline Eryngium carlinae Delar. f. & BP-E & HER \\
\hline Micropleura renifolia Lag. & BP-E & HER \\
\hline Prionosciadium nelsonii J.M. Coult. \& Rose & BP-E & HER \\
\hline \multicolumn{3}{|l|}{ APOCYNACEAE } \\
\hline Asclepias auriculata Kunth & BP-E & HER \\
\hline A. linaria Cav. & BP-E & HER \\
\hline Cynanchum foetidum (Cav.) Kunth & BP-E & HER \\
\hline Gonolobus uniflorus Kunth & BP-E & HER \\
\hline Mandevilla holosericea (Sessé \& Moc.) J.K. Williams & BP-E & HER \\
\hline Plumeria rubra L. & BTC & $\mathrm{AB}$ \\
\hline Thenardia floribunda Kunth & BMM, BP-E & HER \\
\hline \multicolumn{3}{|l|}{ APODANTHACEAE } \\
\hline Pilostyles thurberi A. Gray & BP-E & PAR \\
\hline \multicolumn{3}{|l|}{ ARALIACEAE } \\
\hline Oreopanax peltatus Linden & BP-E & $\mathrm{AB}, \mathrm{AR}$ \\
\hline
\end{tabular}


Apéndice 1. Continuación.

\begin{tabular}{|c|c|c|}
\hline Especie & Vegetación & $\begin{array}{l}\text { Forma } \\
\text { de vida }\end{array}$ \\
\hline \multicolumn{3}{|l|}{ ASTERACEAE } \\
\hline Ageratina mairetiana (DC.) R.M. King \& H. Rob. & BP-E & $\mathrm{AB}$ \\
\hline A. pazcuarensis (Kunth) R.M. King \& H. Rob. & BP-E & HER \\
\hline A. petiolaris (Moc. ex DC.) R.M. King \& H. Rob. & BP-E & $\mathrm{AB}$ \\
\hline Ageratina sp. & BP-E & $\mathrm{AB}$ \\
\hline Ageratum corymbosum Zuccagni & BP-E, BTC & HER \\
\hline Alloispermum integrifolium (DC.) H. Rob. & BP-E & $\mathrm{AB}$ \\
\hline A. scabrum (Lag.) H. Rob. & BP-E & HER \\
\hline Alomia alata Hemsl. & BP-E & HER \\
\hline Archibaccharis schiedeana (Benth.) J.D. Jacks. & BMM & $\mathrm{AB}$ \\
\hline A. serratifolia (Kunth) S.F. Blake & BMM & $\mathrm{AB}$ \\
\hline Aster subulatus Michx. & BP-E, BTC & HER \\
\hline Brickellia pendula (Schrad.) A. Gray & BP-E & $\mathrm{AB}$ \\
\hline Cosmos crithmifolius Kunth & BP-E & HER \\
\hline C. ocellatus Greenm. & BP-E & HER \\
\hline C. scabiosoides Kunth & BP-E & HER \\
\hline Dahlia coccinea Cav. & BP-E & HER \\
\hline Erigeron karvinskianus DC. & BP-E & HER \\
\hline Galinsoga parviflora Cav. & BP-E & HER \\
\hline *Gnaphalium canescens DC. & BP-E & HER \\
\hline G. inornatum DC. & BP-E & HER \\
\hline G. semiamplexicaule DC. & BP-E & HER \\
\hline Iostephane heterophylla (Cav.) Benth. & BP-E & HER \\
\hline Jaegeria hirta (Lag.) Less. & BP-E & HER \\
\hline Lagascea rigida (Cav.) Stuessy & BP-E, BTC & $\mathrm{AB}$ \\
\hline L. helianthifolia Kunth & BP-E, BTC & $\mathrm{AB}$ \\
\hline Lasianthaea helianthoides DC. & BP-E & HER \\
\hline Melampodium divaricatum (Rich.) DC. & BP-E, BTC & HER \\
\hline M. microcephalum Less. & BP-E & HER \\
\hline Montanoa frutescens Mairet ex DC. & BMM & $\mathrm{AB}$ \\
\hline Pittocaulon praecox (Cav.) H. Rob. \& Brettell & BTC & $\mathrm{AB}$ \\
\hline Piqueria pilosa Kunth & BP-E & HER \\
\hline Porophyllum viridiflorum (Kunth) DC. & BP-E & HER \\
\hline Roldana angulifolia (DC.) H. Rob. \& Brettell & BP-E & $\mathrm{AB}$ \\
\hline R. lobata La Llave & BP-E, BTC & $\mathrm{AB}$ \\
\hline Rumfordia floribunda DC. & BP-E & $\mathrm{AB}$ \\
\hline Sabazia humilis (Kunth) Cass. & BP-E & HER \\
\hline
\end{tabular}


Apéndice 1. Continuación.

\begin{tabular}{|c|c|c|}
\hline Especie & Vegetación & $\begin{array}{l}\text { Forma } \\
\text { de vida }\end{array}$ \\
\hline Senecio mulgediifolius S. Schauer & BP-E & HER \\
\hline Stevia incognita Grashoff & BP-E & HER \\
\hline S. micrantha Lag. & BP-E & HER \\
\hline S. origanoides Kunth & BP-E & HER \\
\hline S. subpubescens Lag. & BP-E, BTC & $\mathrm{AB}$ \\
\hline Steviopsis adenosperma (Sch. Bip.) B.L. Turner & BP-E & HER \\
\hline Tagetes filifolia Lag. & BP-E & HER \\
\hline T. lucida Cav. & BP-E & HER \\
\hline T. lunulata Ortega & BP-E, BTC & HER \\
\hline Tithonia tubiformis (Jacq.) Cass. & BP-E & HER \\
\hline Verbesina tetraptera (Ortega) A. Gray & BP-E & HER \\
\hline Vernonia alamanii DC. & BP-E & $\mathrm{AB}$ \\
\hline V. salicifolia (Mart.) Less. & BP-E & $\mathrm{AB}$ \\
\hline Zinnia peruviana L. & BP-E, BTC & HER \\
\hline \multicolumn{3}{|l|}{ BEGONIACEAE } \\
\hline Begonia biserrata Lindl. & BP-E & HER \\
\hline B. gracilis Kunth & BP-E & HER \\
\hline \multicolumn{3}{|l|}{ BIGNONIACEAE } \\
\hline Distictis buccinatoria (DC.) A.H. Gentry & BMM, BP-E & $\mathrm{AB}$ \\
\hline \multicolumn{3}{|l|}{ BORAGINACEAE } \\
\hline Tournefortia hirsutissima L. & BMM & HER \\
\hline Nama origanifolia Kunth & BP-E & HER \\
\hline Bursera cuneata (Schltdl.) Engl. & BP-E, BTC & AR \\
\hline B. fagaroides (Kunth) Engl. & BP-E, BTC & AR \\
\hline B. glabrifolia (Kunth) Engl. & BP-E, BTC & AR \\
\hline B. longipes (Rose) Standl. & BP-E, BTC & AR \\
\hline \multicolumn{3}{|l|}{ CACTACEAE } \\
\hline Mammillaria spinosissima Lem. & BP-E & HER \\
\hline Nopalea sp. & BTC & $\mathrm{AB}$ \\
\hline Opuntia tomentosa Salm-Dyck & BTC & $\mathrm{AB}$ \\
\hline \multicolumn{3}{|l|}{ CAMPANULACEAE } \\
\hline Diastatea tenera (A. Gray) McVaugh & BTC & HER \\
\hline Lobelia laxiflora Kunth & BP-E & HER \\
\hline \multicolumn{3}{|l|}{ CARYOPHYLLACEAE } \\
\hline Arenaria lanuginosa (Michx.) Rohrb. & BP-E & HER \\
\hline Stellaria cuspidata Willd. ex Schltdl. & BP-E & HER \\
\hline
\end{tabular}


Apéndice 1. Continuación.

\begin{tabular}{|c|c|c|}
\hline Especie & Vegetación & $\begin{array}{c}\text { Forma } \\
\text { de vida }\end{array}$ \\
\hline
\end{tabular}

CELASTRACEAE

Celastrus pringlei Rose

Hippocratea celastroides Kunth

$\begin{array}{cc}\text { BMM } & \text { AB } \\ \text { BMM, BTC } & \text { AB }\end{array}$

CONVOLVULACEAE

Cuscuta corymbosa Ruiz \& Pav.

C. gracillima Engelm.

C. tinctoria Mart. ex Engelm.

Evolvulus alsinoides (L.) L.

Ipomoea alba $\mathrm{L}$.

I. batatas (L.) Lam.

I. bracteata Cav.

I. conzattii Geenm.

I. dimorphophylla Greenm.

I. purga (Wender.) Hayne

I. purpurea (L.) Roth

CRASSULACEAE

Echeveria grandiflora Haw.

E. waltheri Moran \& Meyran

Sedum cremnophila R.T. Clause

S. jaliscanum S. Watson

S. oxypetalum Kunth

S. stelliforme S.Watson

BP-E

PAR

BP-E PAR

BP-E PAR

BTC HER

BTC HER

BTC HER

BTC HER

BP-E, BTC HER

BP-E HER

BP-E HER

BP-E HER

Sedum sp.

CUCURBITACEAE

Cyclanthera ribiflora (Schltdl.) Cogn.

BP-E HER

BP-E HER

BP-E HER

BP-E HER

BP-E AB

BP-E HER

BP-E HER

C. tamnoides (Willd.) Cogn.

Echinopepon gemellus (Ser.) A.K. Monro \& P.J. Stafford

Polyclathra cucumerina Bertol.

BP-E, BTC HER

BMM, BTC HER

BP-E, BTC HER

EBENACEAE

Diospyros digyna Jacq.

BMM, BTC HER

ERICACEAE

Arbutus xalapensis Kunth

BTC

AR

EUPHORBIACEAE

Acalypha mollis Kunth

BP-E

AR

Euphorbia graminea var. novogaliciana McVaugh

BP-E

HER

E. multiseta Benth.

BP-E HER

E. pulcherrima Willd. ex Klotzsch

BP-E

HER

BTC

AB 
Apéndice 1. Continuación.

\begin{tabular}{|c|c|c|}
\hline Especie & Vegetación & $\begin{array}{l}\text { Forma } \\
\text { de vida }\end{array}$ \\
\hline E. schlechtendalii Boiss. & BTC & $\mathrm{AB}$ \\
\hline E. sphaerorhiza Benth. & BP-E & HER \\
\hline \multicolumn{3}{|l|}{ FABACEAE } \\
\hline Acacia acatlensis Benth. & BP-E & AR \\
\hline A. angustifolia (Lam.) Desf. & BP-E, BTC & AR \\
\hline Aeschynomene americana $\mathrm{L}$. & BP-E, BTC & HER \\
\hline Calliandra grandiflora (L'Hér.) Benth. & BP-E, BTC & HER \\
\hline Canavalia villosa Benth. & BP-E, BTC & HER \\
\hline *Clitoria mexicana Link & BMM, BP-E & HER \\
\hline${ }^{*}$ C. polystachya Benth. & BMM, BP-E & HER \\
\hline Cologania broussonetii (Balb.) DC. & BP-E, BTC & HER \\
\hline C. jaliscana $\mathrm{S}$. Watson & BMM, BP-E & HER \\
\hline Crotalaria cajanifolia Kunth & BP-E, BTC & HER \\
\hline C. mollicula Kunth & BP-E, BTC & HER \\
\hline C. sagittalis $\mathrm{L}$. & BP-E, BTC & HER \\
\hline Dalea sericea Lag. & BP-E & HER \\
\hline Desmodium aff. aparines (Link) DC. & BMM, BP-E & HER \\
\hline D. guadalajaranum $\mathrm{S}$. Watson & BMM, BP-E & HER \\
\hline D. intortum (Mill.) Urb. & BP-E & HER \\
\hline D. jaliscanum S. Watson & BMM, BP-E & HER \\
\hline D. molliculum (Kunth) DC. & BMM, BP-E & HER \\
\hline D. skinneri Benth. ex Hemsl. & BP-E & HER \\
\hline Diphysa racemosa Rose & BP-E & $\mathrm{AB}$ \\
\hline Erythrina breviflora Sessé \& Moc. ex DC. & BP-E & $\mathrm{AB}$ \\
\hline Eysenhardtia polystachya (Ortega) Sarg. & BP-E, BTC & AR \\
\hline Galactia multiflora B.L. Rob. & BMM, BP-E & HER \\
\hline Indigofera miniata Ortega & BP-E, BTC & HER \\
\hline Leucaena diversifolia (Schltdl.) Benth. & BP-E, BTC & AR \\
\hline Lupinus mexicanus Cerv. ex Lag. & BP-E & HER \\
\hline Lysiloma acapulcense (Kunth) Benth. & BP-E, BTC & AR \\
\hline Marina nutans (Cav.) Barneby & BMM, BP-E & HER \\
\hline Mimosa albida Humb. \& Bonpl. ex Willd. & BP-E, BTC & $\mathrm{AB}$ \\
\hline M. benthamii J.F. Macbr. & BP-E, BTC & $\mathrm{AB}$ \\
\hline M. caerulea Rose & BP-E, BTC & $\mathrm{AB}$ \\
\hline M. lacerata Rose & BP-E, BTC & $\mathrm{AB}$ \\
\hline Phaseolus coccineus L. & BP-E, BTC & HER \\
\hline P. pedicellatus Benth. & BP-E, BTC & HER \\
\hline
\end{tabular}


Apéndice 1. Continuación.

\begin{tabular}{|c|c|c|}
\hline Especie & Vegetación & $\begin{array}{l}\text { Forma } \\
\text { de vida }\end{array}$ \\
\hline Rhynchosia discolor M. Martens \& Galeotti & BMM, BP-E & HER \\
\hline Senna skinneri (Benth.) H.S. Irwin \& Barneby & BP-E, BTC & $\mathrm{AB}$ \\
\hline S. holwayana (Rose) H.S. Irwin \& Barneby & BP-E, BTC & $\mathrm{AB}$ \\
\hline Zornia thymifolia Kunth & BP-E, BTC & HER \\
\hline \multicolumn{3}{|l|}{ FAGACEAE } \\
\hline Quercus candicans Née & BMM, BP-E & AR \\
\hline Q. castanea Née & BP-E & $\mathrm{AR}$ \\
\hline Q. glaucoides M. Martens \& Galeotti & BP-E & $\mathrm{AR}$ \\
\hline Q. obtusata Bonpl. & BMM, BP-E & AR \\
\hline Q. splendens Née & BP-E & AR \\
\hline \multicolumn{3}{|l|}{ GARRYACEAE } \\
\hline Garrya longifolia Rose & BMM & AR \\
\hline \multicolumn{3}{|l|}{ GERANIACEAE } \\
\hline Geranium schiedeanum Schltdl. & BP-E & HER \\
\hline \multicolumn{3}{|l|}{ GESNERIACEAE } \\
\hline Achimenes grandiflora (Schltdl.) DC. & BP-E & HER \\
\hline A. antirrhina (DC.) C.V. Morton & BP-E & HER \\
\hline \multicolumn{3}{|l|}{ HYPERICACEAE } \\
\hline \multicolumn{3}{|l|}{ LAMIACEAE } \\
\hline Salvia lavanduloides Kunth & BP-E & HER \\
\hline S. leucantha Cav. & BP-E & HER \\
\hline S. mexicana L. & BP-E & HER \\
\hline S. mocinoi Benth. & BP-E & HER \\
\hline S. sessei Benth. & BP-E & $\mathrm{AB}$ \\
\hline Salvia sp. & BP-E & HER \\
\hline Stachys coccinea Ortega & BP-E & HER \\
\hline \multicolumn{3}{|l|}{ LAURACEAE } \\
\hline Litsea glaucescens Kunth & BMM, BP-E & $\mathrm{AB}$ \\
\hline Nectandra salicifolia (Kunth) Nees & BMM, BTC & AR \\
\hline \multicolumn{3}{|l|}{ LENTIBULARIACEAE } \\
\hline Pinguicula moranensis var. moranensis Kunth & BP-E & HER \\
\hline P. moranensis var. neovolcanica Zamudio & BP-E & HER \\
\hline \multicolumn{3}{|l|}{ LOASACEAE } \\
\hline Gronovia longiflora Rose & BMM, BTC & HER \\
\hline \multicolumn{3}{|l|}{ LOGANIACEAE } \\
\hline Spigelia speciosa Kunth & BP-E & HER \\
\hline
\end{tabular}


Apéndice 1. Continuación.

\begin{tabular}{|c|c|c|}
\hline Especie & Vegetación & $\begin{array}{l}\text { Forma } \\
\text { de vida }\end{array}$ \\
\hline \multicolumn{3}{|l|}{ LORANTHACEAE } \\
\hline Cladocolea oligantha (Standl. \& Steyerm.) Kuijt & BP-E & PAR \\
\hline \multicolumn{3}{|l|}{ LYTHRACEAE } \\
\hline Cuphea angustifolia Jacq. ex Koehne & BP-E & HER \\
\hline C. hookeriana Walp. & BP-E & HER \\
\hline C. lanceolata W.T. Aiton & BP-E & HER \\
\hline C. wrightii A.Gray & BP-E & HER \\
\hline \multicolumn{3}{|l|}{ MALPIGHIACEAE } \\
\hline Gaudichaudia diandra Nied. & BTC & HER \\
\hline Mascagnia dipholiphylla (Small) Bullock & BP-E & $\mathrm{AB}$ \\
\hline \multicolumn{3}{|l|}{ MALVACEAE } \\
\hline Abutilon ellipticum Schltdl. & BP-E, BTC & HER \\
\hline Malvaviscus arboreus Cav. & BP-E, BTC & AR \\
\hline Periptera punicea (Lag.) DC. & BMM, BP-E & HER \\
\hline Triumfetta semitriloba Jacq. & BMM, BP-E & HER \\
\hline \multicolumn{3}{|l|}{ MELIACEAE } \\
\hline Guarea glabra Vahl & BMM & AR \\
\hline \multicolumn{3}{|l|}{ MORACEAE } \\
\hline Ficus cotinifolia Kunth & BTC & AR \\
\hline F. petiolaris Kunth & BTC & AR \\
\hline \multicolumn{3}{|l|}{ MYRSINACEAE } \\
\hline Ardisia compressa Kunth & BMM, BP-E, BTC & AR \\
\hline Myrsine juergensenii (Mez) Ricketson \& Pipoly & BMM & AR \\
\hline \multicolumn{3}{|l|}{ MYRTACEAE } \\
\hline Eugenia crenularis Lundell & BMM & AR \\
\hline \multicolumn{3}{|l|}{ OLEACEAE } \\
\hline Fraxinus uhdei (Wenz.) Lingelsh. & BMM & AR \\
\hline \multicolumn{3}{|l|}{ ONAGRACEAE } \\
\hline Fuchsia arborescens Sims & BP-E & $\mathrm{AB}$ \\
\hline F. decidua Standl. & BP-E & $\mathrm{AB}$ \\
\hline F. encliandra Steud. & BP-E & $\mathrm{AB}$ \\
\hline F. fulgens DC. & BP-E & $\mathrm{AB}$ \\
\hline Gongylocarpus rubricaulis Schltdl. \& Cham. & BP-E & HER \\
\hline Lopezia longiflora Decne. & BMM, BP-E & $\mathrm{AB}$ \\
\hline L. miniata Lag. ex DC. & BP-E & HER \\
\hline Ludwigia octovalvis (Jacq.) P.H. Raven & BP-E, BTC & HER \\
\hline
\end{tabular}


Apéndice 1. Continuación.

\begin{tabular}{l}
\hline Especie \\
\hline OPILIACEAE \\
Agonandra racemosa (DC.) Standl. \\
OROBANCHACEAE \\
Castilleja arvensis Schltdl. \& Cham. \\
C. tenuiflora Benth. \\
C. tenuifolia M. Martens \& Galeotti \\
Conopholis alpina Liebm. \\
Escobedia grandiflora (L.f.) Kuntze \\
Lamourouxia longiflora Benth.
\end{tabular}

L. multifida Kunth Vegetación

Forma

de vida

OXALIDACEAE

Oxalis jacquiniana Kunth

BP-E AR

O. latifolia Kunth

O. lunulata Zucc.

BP-E

HER

BP-E

HER

BP-E

HER

BP-E

PAR

BP-E

HER

BP-E

HER

BP-E

HER

O. tetraphylla Cav.

Oxalis sp.

BP-E

HER

BP-E

HER

BP-E

HER

BP-E

HER

PASSIFLORACEAE

Passiflora suberosa L.

BP-E

HER

Passiflora sp.

BP-E

HER

PHRYMACEAE

Mimulus glabratus Kunth

BP-E

HER

PLANTAGINACEAE

Lophospermum scandens D. Don

BP-E, BTC HER

POLEMONIACEAE

Loeselia glandulosa (Cav.) G.Don

L. mexicana (Lam.) Brand

BP-E

HER

POLYGALACEAE

Monnina ciliolata Sessé \& Moc. ex DC.

Polygala appressipilis S.F.Blake

$P$. paniculata L.

BP-E

HER

BP-E HER

Polygala sp.

BMM, BP-E

AB

BP-E

HER

BP-E

HER

BP-E

HER

POLYGONACEAE

Polygonum amphibium L.

BTC

HER

RANUNCULACEAE

Clematis dioica L.

BP-E

AB

Delphinium pedatisectum Hemsl.

BP-E

HER

Thalictrum gibbosum Lecoy.

BP-E

HER 
Apéndice 1. Continuación.

\begin{tabular}{|c|c|c|}
\hline Especie & Vegetación & $\begin{array}{l}\text { Forma } \\
\text { de vida }\end{array}$ \\
\hline RHAMNACEAE & & \\
\hline $\begin{array}{l}\text { Frangula hintonii (M.C. Johnst. \& L.A. Johnst.) A. Pool } \\
\text { RUBIACEAE }\end{array}$ & BMM & AR \\
\hline Borreria prostrata (Aubl.) Miq. & BTC & HER \\
\hline Bouvardia laevis M. Martens \& Galeotti & BP-E & HER \\
\hline B. multiflora (Cav.) Schult. \& Schult. f. & BP-E & HER \\
\hline B. ternifolia (Cav.) Schltdl. & BP-E & HER \\
\hline Crusea coccinea DC. & BP-E & HER \\
\hline C. longiflora (Willd. ex Roem. \& Schult.) W.R. Anderson & BP-E & HER \\
\hline Hoffmannia cuneatissima B.L. Rob. & BMM & $\mathrm{AB}$ \\
\hline Galium mexicanum Kunth & BP-E & HER \\
\hline *Randia obcordata S. Watson & BTC & $\mathrm{AB}$ \\
\hline R. thurberi S. Watson & BTC & $\mathrm{AB}$ \\
\hline RUTACEAE & & \\
\hline *Amyris balsamifera $\mathrm{L}$. & BMM, BTC & AR \\
\hline *Amyris rekoi Blake & BMM & $\mathrm{AB}$ \\
\hline $\begin{array}{l}\text { Esenbeckia vazquezii Ramos \& E. Martínez } \\
\text { SABIACEAE }\end{array}$ & BMM, BTC & AR \\
\hline $\begin{array}{l}\text { Meliosma dentata (Liebm.) Urb. } \\
\text { SALICACEAE }\end{array}$ & BMM & AR \\
\hline Xylosma flexuosa (Kunth) Hemsl. & BMM, BTC & $\mathrm{AR}$ \\
\hline $\begin{array}{l}\text { Xylosma sp. } \\
\text { SANTALACEAE }\end{array}$ & BMM, BTC & AR \\
\hline Phoradendron brachystachyum (DC.) Nutt. & BP-E & PAR \\
\hline $\begin{array}{l}\text { P. decipiens Kuijt } \\
\text { SAPINDACEAE }\end{array}$ & BTC & PAR \\
\hline $\begin{array}{l}\text { Serjania mexicana (L.) Willd. } \\
\text { SAPOTACEAE }\end{array}$ & BTC & HER \\
\hline $\begin{array}{l}\text { *Sideroxylon cartilagineum (Cronquist) T.D. Penn. } \\
\text { SCROPHULARIACEAE }\end{array}$ & BTC & AR \\
\hline Russelia coccinea (L.) Wettst. & BP-E & HER \\
\hline $\begin{array}{l}\text { Buddleia sessiliflora Kunth } \\
\text { SOLANACEAE }\end{array}$ & BP-E & $\mathrm{AB}$ \\
\hline *Brachistus stramoniifolius (Kunth) Miers & BMM, BTC & $\mathrm{AB}$ \\
\hline Cestrum nitidum M. Martens \& Galeotti & BP-E & $\mathrm{AB}$ \\
\hline C. thyrsoideum Kunth & BP-E & $\mathrm{AB}$ \\
\hline C. tomentosum L. f. & BP-E, BTC & $\mathrm{AB}$ \\
\hline
\end{tabular}


Apéndice 1. Continuación.

\begin{tabular}{|c|c|c|}
\hline Especie & Vegetación & $\begin{array}{l}\text { Forma } \\
\text { de vida }\end{array}$ \\
\hline Cestrum sp. & BP-E & $\mathrm{AB}$ \\
\hline Jaltomata procumbens (Cav.) J.L. Gentry & BP-E & HER \\
\hline *Lycianthes peduncularis (Schltdl.) Bitter & BP-E & HER \\
\hline${ }^{*}$ L. pilosissima (M. Martens \& Galeotti) Bitter & BP-E & HER \\
\hline L. rzedowskii E.A. Dean & BP-E & HER \\
\hline Physalis gracilis Miers & BP-E & HER \\
\hline Solanum cervantesii Lag. & BP-E & $\mathrm{AB}$ \\
\hline S. marginatum L. f. & BP-E & $\mathrm{AB}$ \\
\hline S. nigrescens M. Martens \& Galeotti & BP-E & HER \\
\hline $\begin{array}{l}\text { S. nigricans M. Martens \& Galeotti } \\
\text { STYRACACEAE }\end{array}$ & BP-E & $\mathrm{AB}$ \\
\hline $\begin{array}{l}\text { Styrax ramirezii Greenm. } \\
\text { SYMPLOCACEAE }\end{array}$ & BMM & AR \\
\hline $\begin{array}{l}\text { Symplocos citrea Lex. ex La Llave \& Lex. } \\
\text { THEACEAE }\end{array}$ & BMM & AR \\
\hline $\begin{array}{l}\text { Ternstroemia lineata DC. } \\
\text { ULMACEAE }\end{array}$ & BMM, BP-E & AR \\
\hline $\begin{array}{l}\text { Trema micrantha (L.) Blume } \\
\text { URTICACEAE }\end{array}$ & BMM, BTC & AR \\
\hline Myriocarpa brachystachys S. Watson & BP-E, BTC & AR \\
\hline Parietaria macrophylla B.L. Rob. \& Greenm. & BMM & HER \\
\hline Pilea hyalina Fenzl & BP-E & HER \\
\hline Urera baccifera (L.) Gaudich. ex Wedd. & BMM, BP-E & AR \\
\hline $\begin{array}{l}\text { U. caracasana (Jacq.) Gaudich. ex Griseb. } \\
\text { VALERIANACEAE }\end{array}$ & BMM & AR \\
\hline Valeriana palmeri A. Gray & BP-E & HER \\
\hline V. sorbifolia Kunth & BP-E & HER \\
\hline V. tafiensis Borsini & BP-E & HER \\
\hline $\begin{array}{l}\text { V. urticaefolia Kunth } \\
\text { VERBENACEAE }\end{array}$ & BP-E & HER \\
\hline Lippia mexicana G. L. Nesom & BP-E & HER \\
\hline Citharexylum hintoni Moldenke & BP-E & $\mathrm{AB}$ \\
\hline
\end{tabular}


Acta Botanica Mexicana 108: 11-38 (2014)

\section{APÉNDICE 2}

Flora representativa de la Barranca Tepecapa.
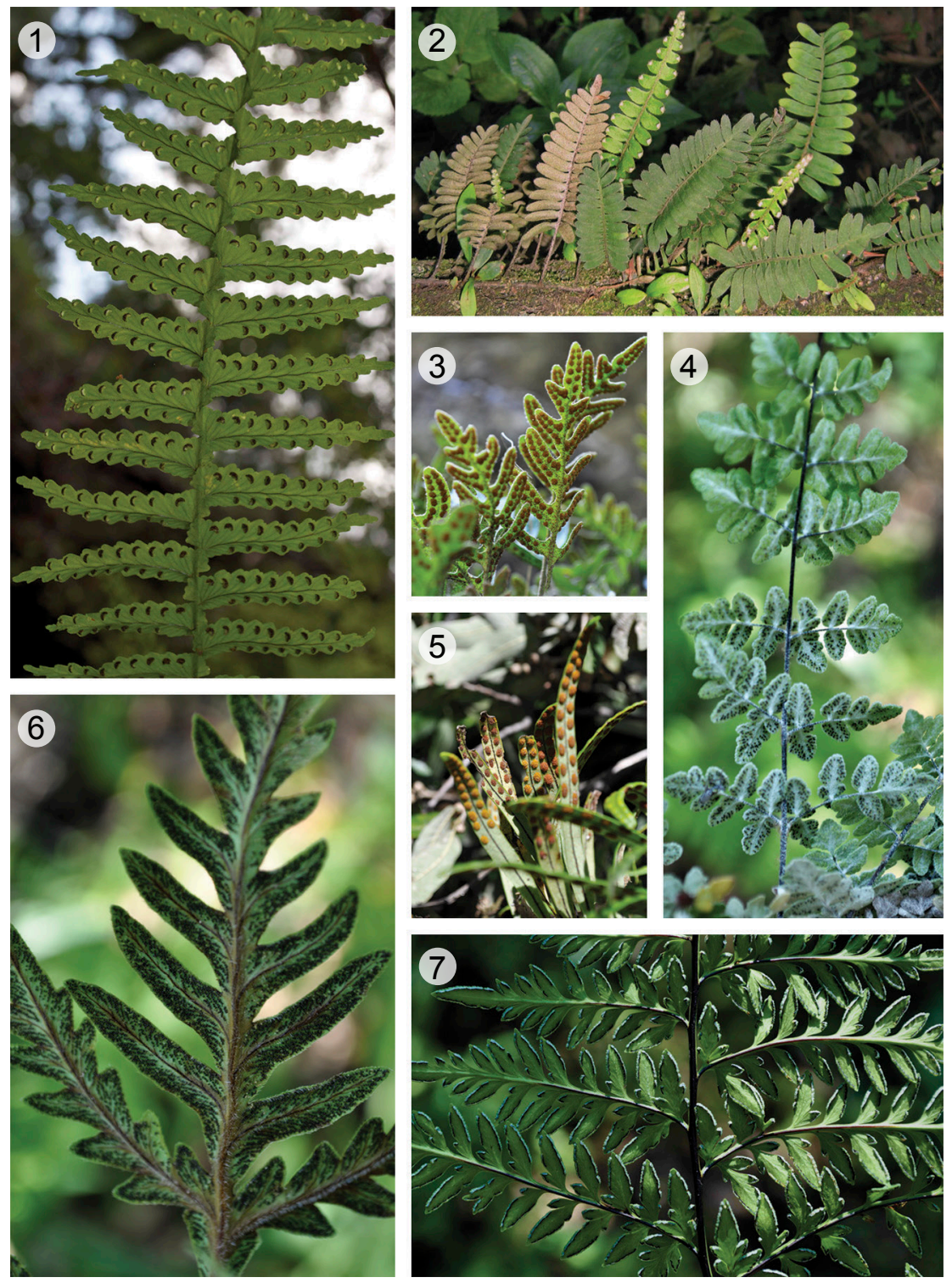

1. Nephrolepis undulata; 2. Polypodium polypodioides; 3. Polypodium thyssanolepis; 4. Argyrochosma pilifera; 5. Pleopeltis polylepis; 6. Bommeria pedata; 7. Cheilanthes cuneata. 
Hernández-Cárdenas et al.: Plantas vasculares y vegetación de la Barranca Tepecapa, Morelos, México
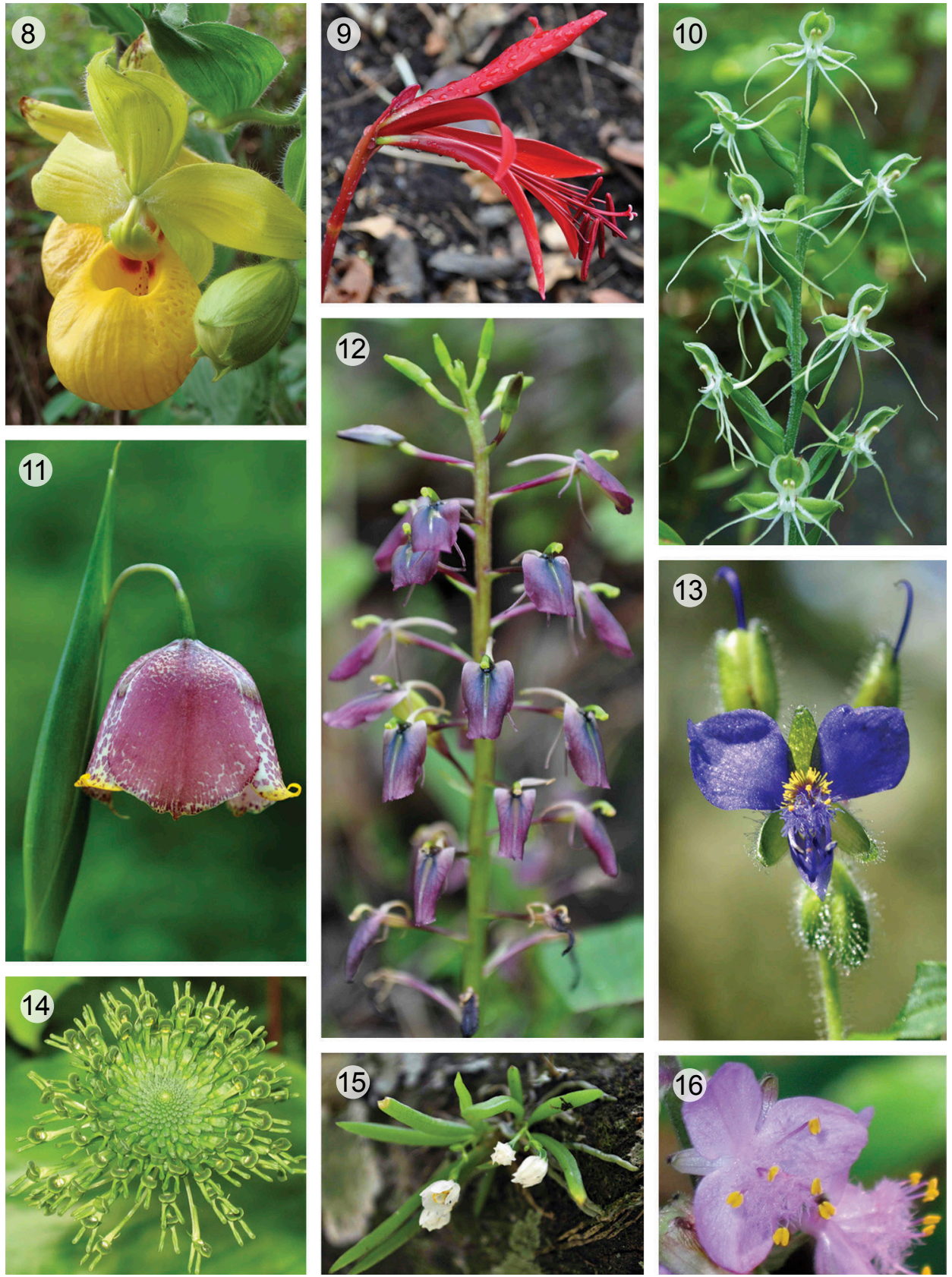

8. Cypripedium irapeanum; 9. Sprekelia formosissima; 10. Habenaria crassicornis; 11. Tigridia meleagris; 12. Liparis greenwoodiana; 13. Tinantia erecta; 14 . Malaxis brachyrrhynchos; 15. Hintonella mexicana; 16. Thyrsanthemum macrophyllum. 

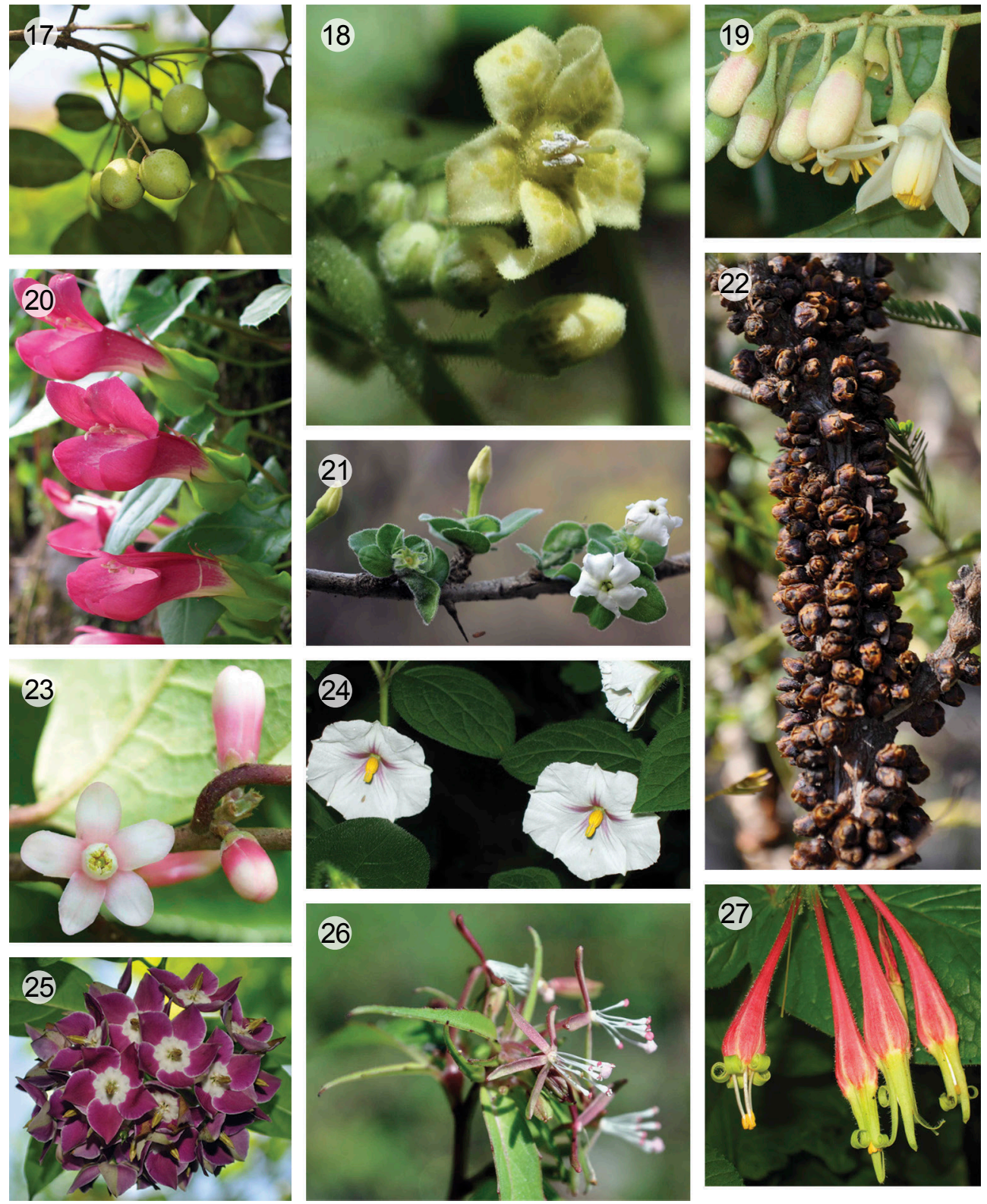

17. Amyris balsamifera; 18. Brachistus stramoniifolius; 19. Styrax ramirezii; 20. Lophospermum scandens; 21. Randia obcordata; 22. Pilostyles thurberi; 23. Symplocos citrea; 24. Lycianthes peduncularis; 25. Thenardia floribunda; 26. Gongylocarpus rubricaulis; 27. Spigelia speciosa. 